\title{
CYCLIC BEHAVIOUR OF BEAM-COLUMN DOWEL CONNECTION IN PRECAST ELEMENTS
}

\author{
MARIUS G.L. MOLDOVAN ${ }^{1}$, MIHAI NEDELCU ${ }^{2}$ AND ZSOLT KOVACS ${ }^{3}$ \\ ${ }^{1}$ TUCN/CONSOLIS \\ Str. Memorandumului 28, Cluj-Napoca 400114, Romania \\ George.Moldovan@mecon.utcluj.ro,www.utcluj.ro \\ 2 TUCN \\ Str. Memorandumului 28, Cluj-Napoca 400114, Romania \\ Mihai.Nedelcu@mecon.utcluj.ro, users.utcluj.ro/ mnedelcu/index_en.htm \\ ${ }^{3}$ CONSOLIS \\ Lajos utca 160-162, 1036 Budapest, Hungary \\ Zsolt.Kovacs@asa.hu
}

Key words: Cyclic loading, seismic behaviour, precast reinforced concrete elements, dowel connection, experimental evaluation.

\begin{abstract}
Disruption of structural continuity due to the inherent nature of the connections poses a challenge in seismic design of precast concrete structures. Seismic behaviour of portal frame systems typically used for industrial halls, is greatly influenced by the beam-column connections. Capacity design dictates that these connections should have an elastic behaviour under seismic horizontal actions to allow for the dissipation mechanisms to develop in the desired area, in this case the base of the columns. If this connection fails, the entire structure is compromised and may lead to a premature, partial, or even total collapse. Efforts are currently underway for a better understanding of the seismic response of precast structures (e.g. SAFECAST project).

In this study, the test setups tried to replicate as closely as possible the behaviour of a commonly used beam-column assembly connected by steel dowels. The test specimens were designed as full scale precast concrete elements. This experimental campaign aimed to determine the failure mechanisms of the assemblies and to check if capacity design requirements were satisfied. Three setups have been tested according to the experimental protocol described in the SAFECAST report. The first test specimen was subjected to a unidirectional monotonic loading protocol with the aim of observing the maximum failure force and deformation. The resulted maximum displacement was used to determine the displacement step increment for the cyclic loading protocol of the following two specimens. In all the cases, failure has occurred in the region of the dowel connection. The failure mechanism was either because of dowel yielding, concrete spalling around the dowel, or a combination of both, consistent with results obtained by other researchers. The results have showed that the column was far from reaching its failure capacity and a premature failure has occurred in the connection area, which should be avoided in common practice.
\end{abstract}




\section{INTRODUCTION}

Precast concrete is defined as the concrete that has been cast and cured in a location that does not coincide with the final position of the element. Precast concrete industry is increasing its share on the construction market worldwide. Controlled manufacturing process (mix, placement, accelerated curing), increased quality, labour efficiency, repeatability, quick assembly, flexibility in use, speed of erection, cost-effectiveness, eco-friendliness and sustainability are the clear advantages of precast when compared with cast-in-place concrete.

Precast elements are joined together using different methods, to form a complete structure. These methods may vary depending on different factors and considering the prescriptions from the codes available in each country; they may range from simple (a simple bearing ledge or corbel) to more complex and harder to execute joints. By using adequate joint connections, it is possible to build a three-dimensional skeleton of the structure that is able to withstand vertical (gravitational) and horizontal (wind and earthquake) loads ${ }^{[1]}$.

Construction in seismic areas becomes everyday more challenging due to the progress of both seismology and seismic engineering. All types of constructions, including those using structural concrete in general and precast concrete in particular, are affected by these developments ${ }^{[2]}$

Although there are many possibilities and solutions in the precast industry, one of the most common systems used for warehouses and industrial halls is the "Portal frame system". The columns are usually clamped in moment resisting foundations and the beams are simply

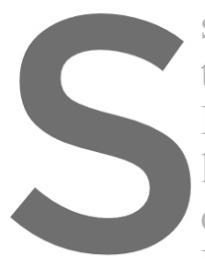
supported on the top of the most problematic In non-seismic practice lateral loads from wind considerations do not fu In recent years, researchers have
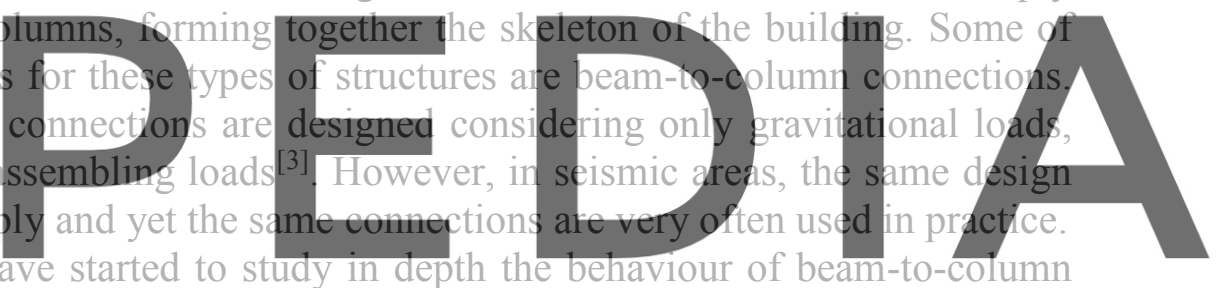
connections in seismic areas. Despite the recent concern regarding this topic the information

of solutions, the major differences being in the case of connections, and information for the behaviour of each individual system during an earthquake event is incomplete and, in some cases, even missing. There are many variations of hinged connections worldwide, but the vast majority use one or two parallel steel dowels that are placed in the direction normal to the plane of the frame. In Europe for example, it is common to use two steel dowels that are anchored in the column and extend through grouted tubes inside the beam ${ }^{[2]}$. One example for this type of connections can be seen in Figure 1. Recent earthquakes from the Italian region of Emilia have provided researchers with in-depth information about the behaviour of reinforced concrete precast industrial buildings. It should be noted that the large majority of the buildings were designed without any concern towards seismic design ${ }^{[4]}$. Some of the buildings that were observed in the area had columns that featured some lateral restraint for the beams in the form of upper pockets (forks) at the top part of the column. These forks were not designed to withstand horizontal actions and they had minimum steel reinforcements; despite this they played an important role in restraining the end rotation of the beam, preventing a possible total collapse. 

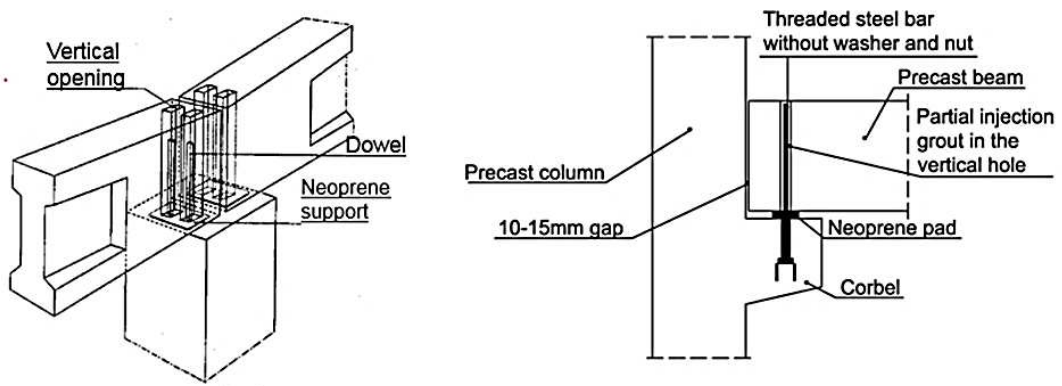

Figure 1: Beam to column connection example

There is a lack of knowledge in Europe in the field of seismic design of precast elements, stressed mostly by the absence of clear design procedures in Eurocode $8^{[5]}$. Efforts are currently underway to better understand the seismic response of precast structures, one of the most known being the SAFECAST project ${ }^{[6]}$. Cast-in-place buildings subjected to seismic loads have been extensively studied in the past years, offering engineers the tools necessary to design resilient structures. On the other hand, precast structures did not get tremendous consideration, and for this reason the design, especially on the subject of the connections, is greatly influenced by local codes and practices. The information on their behaviour during seismic event is limited and even non-existent ${ }^{[7]}$. Based on limited observations, it is challenging to make a general assumption on the behaviour of precast connections during an

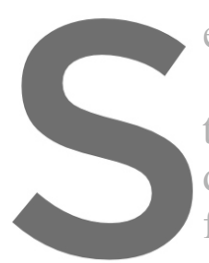
earthquake.

Usually, the design the general aspects, but

details of the connections. Eurocode $8^{[5]}$ of fulfilled for the design of connections; there is

also stated that all connections should be overdesigned by fo
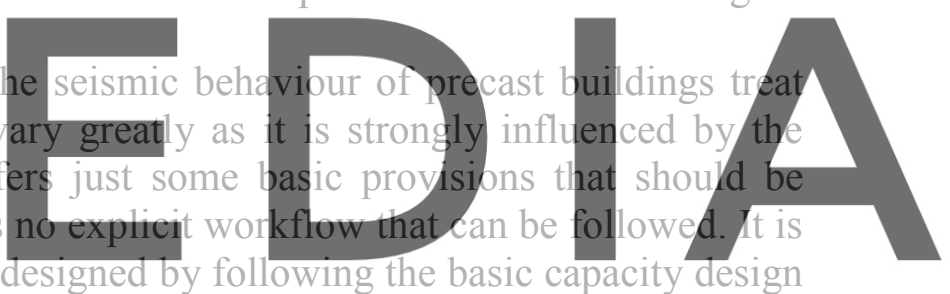
rules, and if the proyisions mentioned do not fully coyer the considered connection, then it is

\section{EXPERIVIENTAL PROGRAIV ${ }^{1}$}

\subsection{Specimens description}

The objective of this research is to investigate the failure mechanism for the proposed assembly. For the experimental program a widely used beam-column assembly was chosen and then adapted to accommodate the entire setup to the laboratory limitations. For this, the length of the column was reduced from $8.5 \mathrm{~m}$ to $6 \mathrm{~m}$. In order to obtain equivalent top displacements and rotations, the cross-section was also reduced from $80 \times 80 \mathrm{~cm}$ to $60 \times 60 \mathrm{~cm}$. The goal was to minimize as much as possible the modifications for the elements used in the tests in order to capture, as accurately as possible, the behaviour of a real-life precast frame structure with beam to column dowel connection. The connection consists of two parallel dowels embedded in the column fork, that are inserted into vertical openings inside the precast beams. Mortar is inserted into these sleeves to ensure the connection the elements.

\footnotetext{
${ }^{1}$ The tests were performed at the ÉMI-TÜV SÜD Kft. Építőipari Szolgáltatások Osztály, Szentendre, Hungary.
} 
Reducing the size of the elements affects the concrete cover for the dowel connection and the results are greatly influenced. In order to avoid this phenomenon, the test specimens crosssections at the joint had dimensions similar to those used in real-life structures. The beam length was approximately $3 \mathrm{~m}$ and it was chosen based on the laboratory limitations and also to avoid any local effect on our connection due to the application of the load at the other end of the beam. Prestress concrete beams can have lengths of over $20 \mathrm{~m}$. The dimensions of the specimens can be seen in Figure 2.

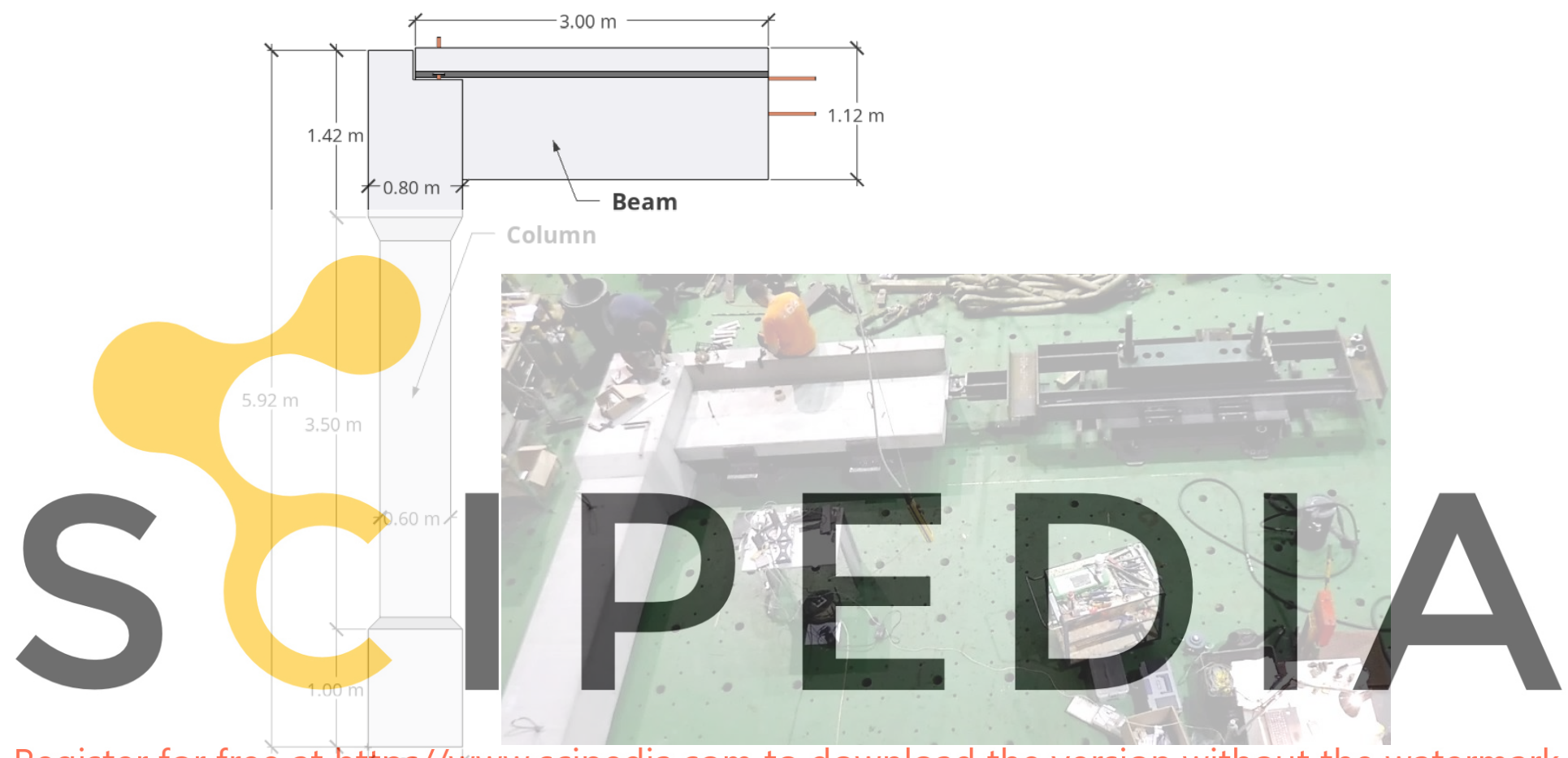

Register for free at https//www.scipedia.com to download the version without the watermark

Figure 2: Specimens dimensions

Because of the large size of the specimens, and safety issues, the tests were performed horizontally on the laboratory floor. The main drawback of this approach is that it induces some additional friction on the sides of the column and the beam that are supported on the floor.

The column $60 \times 60 \mathrm{~cm}$ cross-section was reinforced with longitudinal bars of $25 \mathrm{~mm}$ diameter. For the stirrups $8 \mathrm{~mm}$ diameter bars were positioned at a distance of $100 \mathrm{~mm}$ over the length of the column. The cross-sections at the bottom of the columns were $80 \mathrm{x} 80 \mathrm{~cm}$, with additional reinforcement bars, in order to increase the stiffness of the column in that region; this replicates the effect of the column being clamped at the base in a moment resisting foundation. If failure or development of a plastic hinge occurs in the column this will be driven in the area where the cross-section change happens. The cross-section of the column fork was also $80 \mathrm{x} 80 \mathrm{~cm}$. Height of the beam was $112 \mathrm{~cm}$ and width of the flange $64 \mathrm{~cm}$. At the end of the beam not connected with the column two steel bars were included for fitting the steel assembly that will transmit the load to our specimens. Additional details regarding the 
reinforcement placement is provided in Figure 3 for the column. The dimensions of the elements and the reinforcement percentages were chosen according to real-life design cases and existing structures.

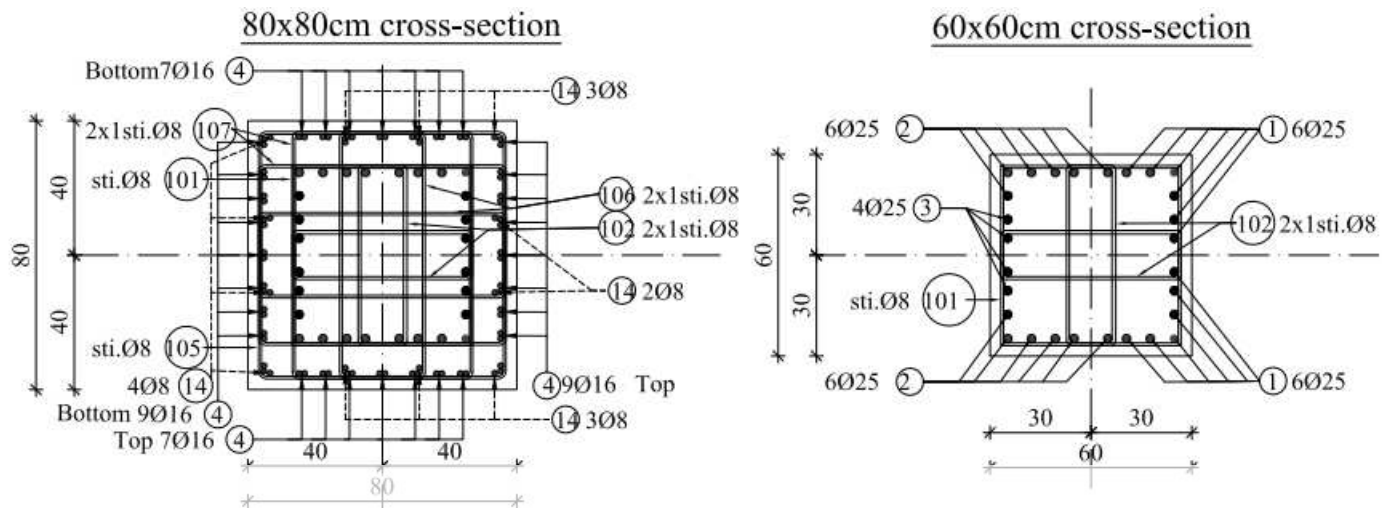

Figure 3: Column cross-section and reinforcement details

Three sets of specimens were casted for this experimental campaign. The first two sets, \#1 and $\# 2$, corresponding to the monotonic test and first cyclic test, respectively, were identical in terms of concrete specimen's dimensions. One notable difference is the dowel diameter: $27 \mathrm{~mm}$ for specimens \#1, and $32 \mathrm{~mm}$ for specimens \#2. For set \#3 the dowel diameter is

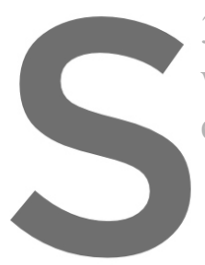
$32 \mathrm{~mm}$, but the thickness web was increased acc concrete coverage of
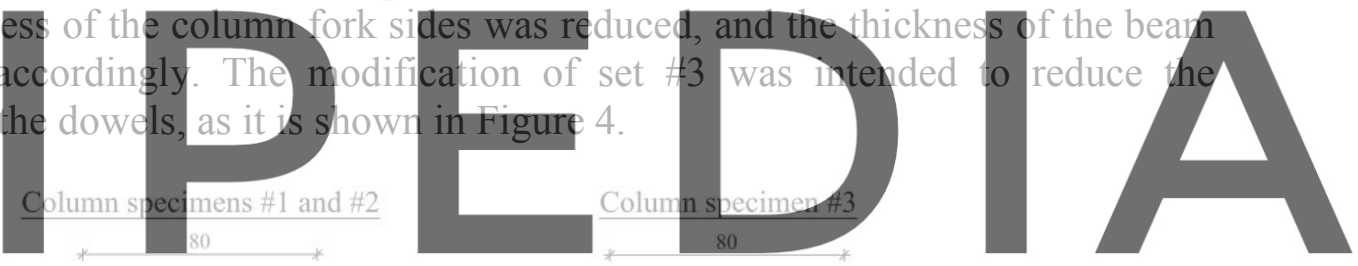

Register for free at https//wWw:sevedra.eom to downfoad the version without the watermark

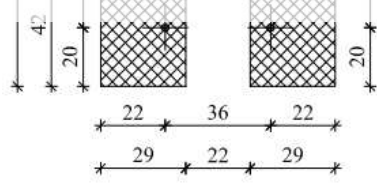

Beam specimens \#1 and \#2

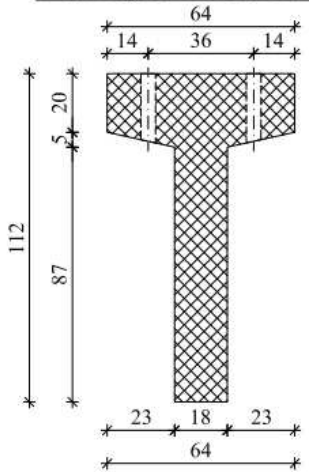

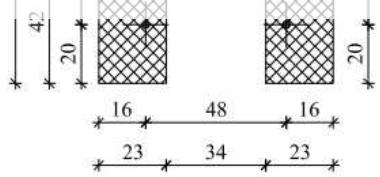

Beam specimen \#3

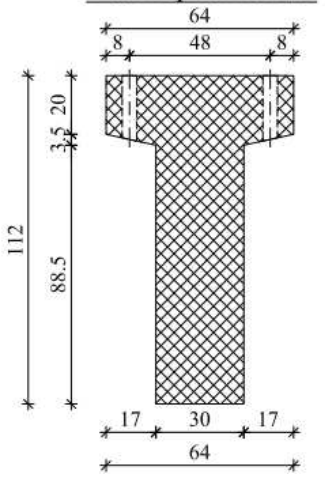

Figure 4: Column and beam cross-sections - differences between specimens \#1, \#2 and \#3 


\subsection{Material properties}

Table 1 presents the material properties determined on concrete specimens according to the Eurocode provisions ${ }^{[9,10,11]}$ on the day the tests were performed: compressive strength (for both cube and cylinder), tensile strength, modulus of elasticity, strain at maximum force. The stress-strain curves were also determined using optical methods.

As one can observe from Table 1, high strength concrete was used for both column and beam. Usually in normal practice, prestressed beams use such a high class of concrete (C50/60) and the columns are usually casted using a C35/45 concrete class. Because each set of specimens were casted in different days samples were taken for each set plus two extra sets of specimens for control. For all the reinforcements, including the dowels, S500B steel class was used S500B steel class (average steel properties: $f_{y}=561 \mathrm{MPa}, f_{\max }=656 \mathrm{MPa}$, $\left.\varepsilon_{\max }=10.73 \%\right)$.

Table 1: Average concrete properties

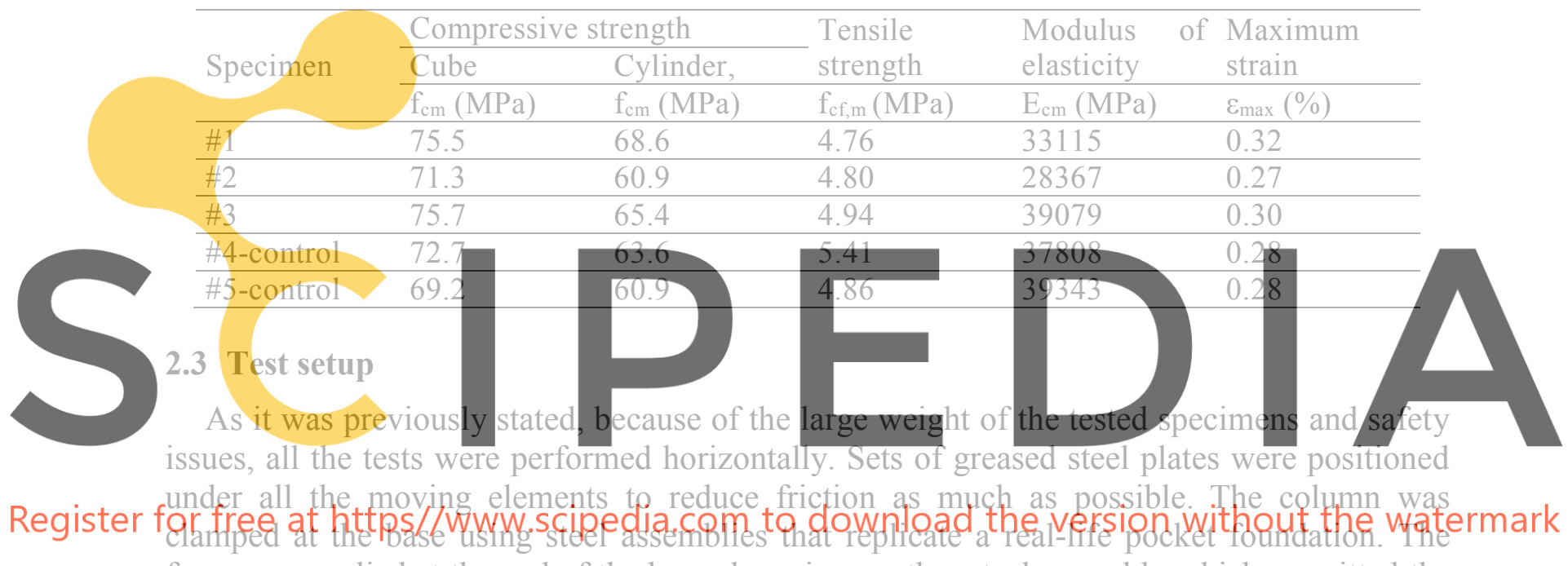

force was applied at the end of the beam by using another steel assembly which permitted the installation of the hydraulic jack, from one side to another, to perform the cyclic analysis.

Steel supports for the beam and the force transmitting frame maintained the direction when applying the load. All the steel assemblies were custom made for these tests. The details of the test setup can be seen in Figure 5 and Figure 6 . All the steel assemblies were fixed in the strong floor of the laboratory.

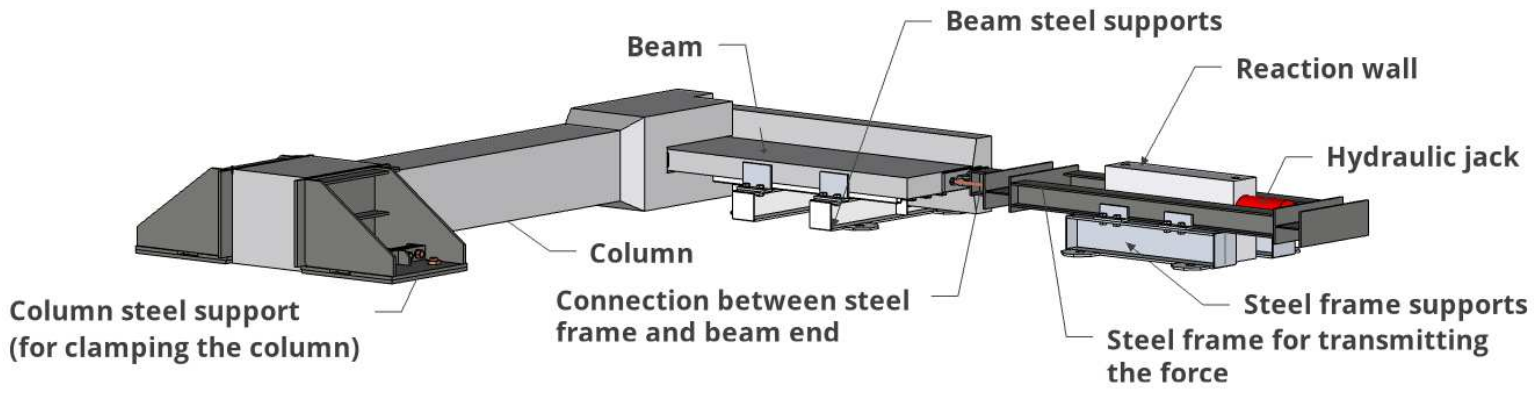

Figure 5: Test setup description - Perspective view 


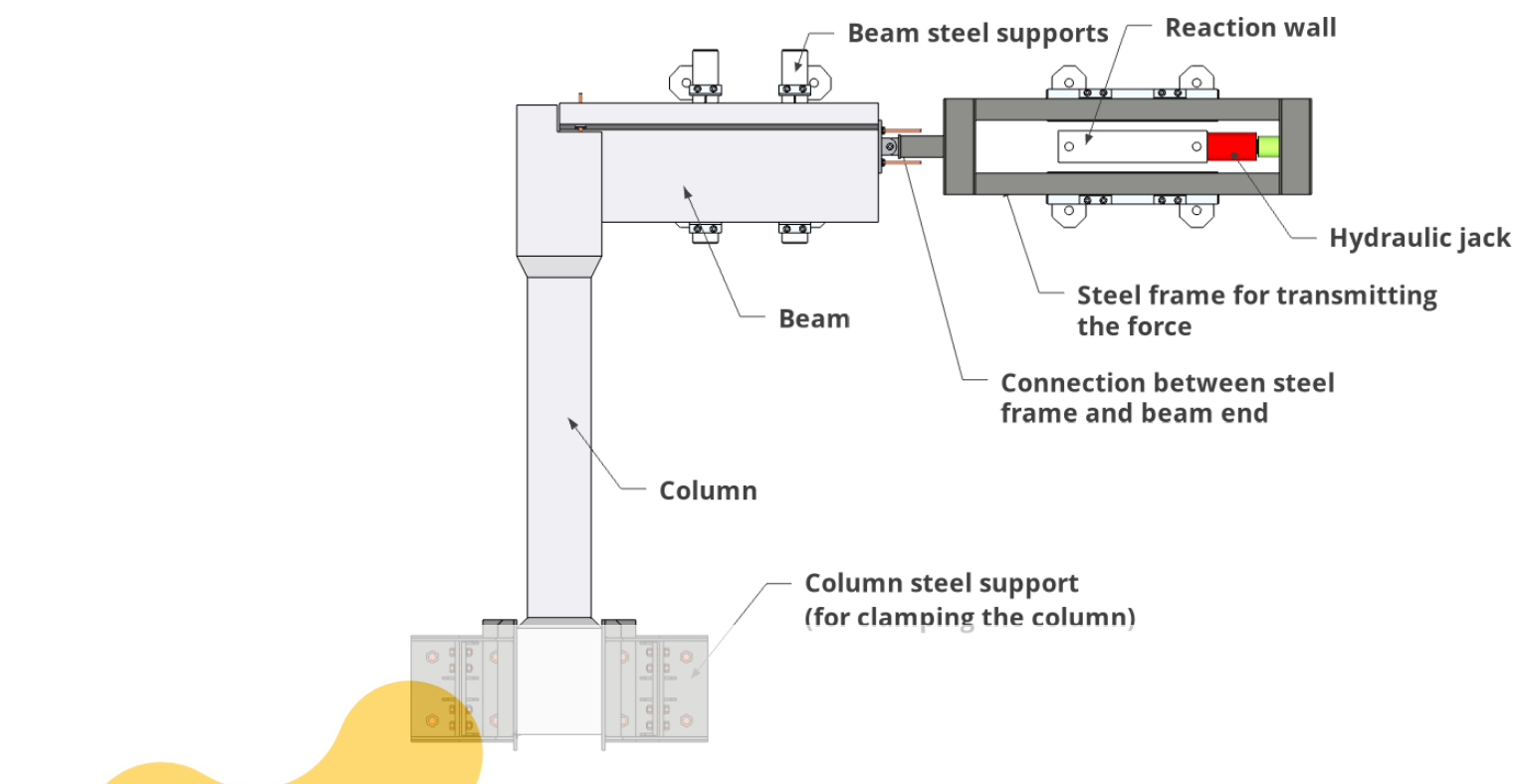

Figure 6: Test setup description - Top view

The connection between the two concrete elements was done by injecting the sleeves inside the beam with high performance anchoring adhesive. The product used was Sika AnchorFix $(\mathbb{R}-3030$, which has a compressive strength of $95 \mathrm{MPa}$, and a tensile strength $23 \mathrm{MPa}{ }^{[8]}$. After curin

Loading was perform

The maximum displacement (at failure) was experimental cyclic response was obtained by applying the load history described meaning groups of three cycles of the same amplitude are performed step by step with

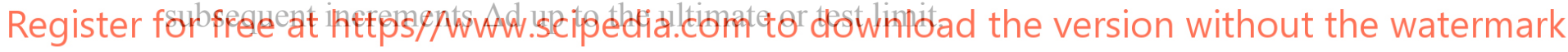

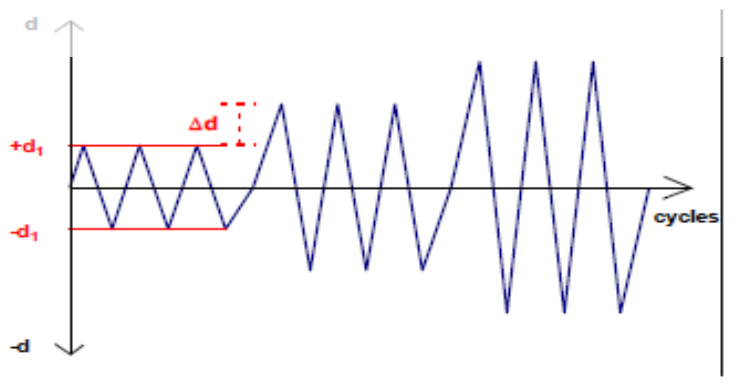

Figure 7: Loading history of the cyclic test ${ }^{[6]}$

During the experiments, the lateral force was continuously measured to obtain the forcedisplacement diagrams. In addition, displacements transducers and strains gauges were installed in critical points (Figure 8). This information is essential for calibrating later, the finite element numerical models. After each cycle, the crack openings on the surface of the column and beam elements were measured. 


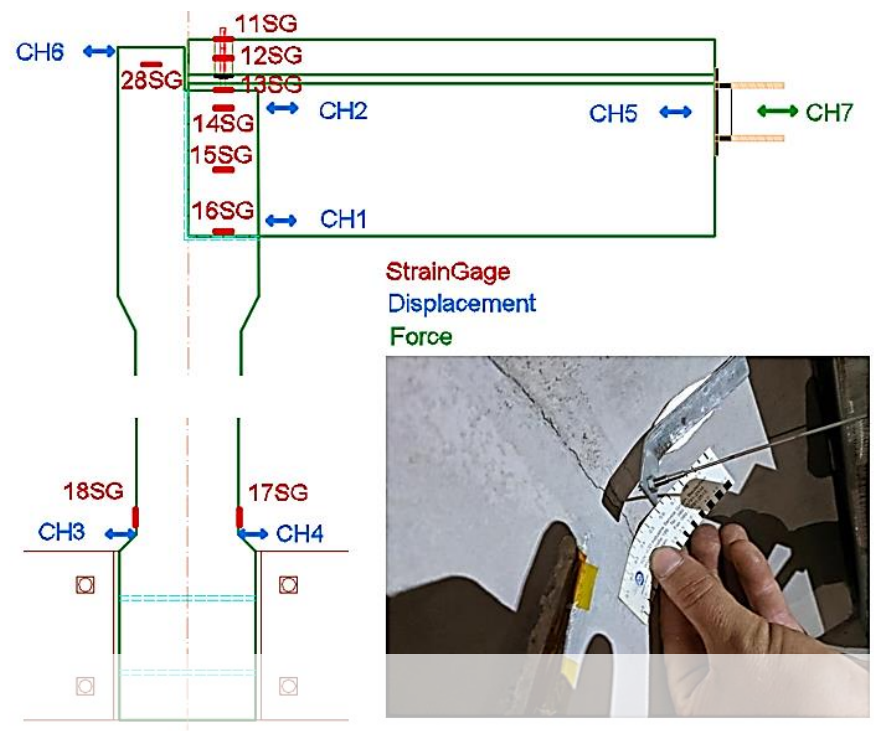

Figure 8: Positions for the measuring equipment

\section{EXPERIMENTAL RESULTS}

For the first set of specimens (\#1), a monotonic test was performed. This was done by pulling the end of the beam, this being the most unfavourable situation for this connection. The whole bearing capacity is given by the shear canacity of the two dovels combined with a low concrete coverage top of the shear strength due to the dowel catenary effect. For test specimens \#2 and determined based on the maximum displacement obtained at f

This displacement was divided into three equal increments. The force-displacement oraphs

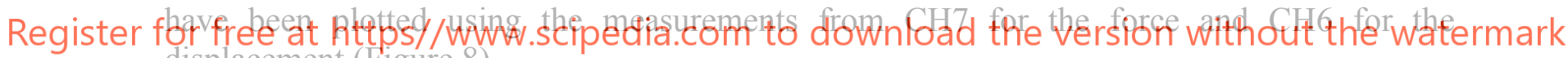
displacement (Figure 8).

\subsection{Results for test specimens \#1 - Monotonic test}

This first test was force controlled with a $\Delta \mathrm{d}=10 \mathrm{kN}$ increment at each step until failure. After each step, the cracks and the connection were checked. Failure was reached at approximately $208 \mathrm{kN}$ with a maximum displacement of $182 \mathrm{~mm}$ (Figure 9). It should be noted that the displacement is much larger than expected because the column base support allowed for some rotation and the column was not completely fixed. This rotation will be calculated and extracted later for obtaining the absolute displacement. Because of the configuration of the testing setup, it is nearly impossible to completely eliminate the friction between the structural concrete elements and the supports. Friction force was shown to be at around $30 \mathrm{kN}$. Kinetic friction was constant over the test duration.

Above the $120 \mathrm{kN}$ point, the steel dowel started deforming. Yielding of the bar was reached at the force of $208.7 \mathrm{kN}$ which coincides with the failure point of the connection. There was also some small crushing of the concrete around the dowel (Figure 10). Most probably this would have increased but the test was stopped due to safety reasons and to avoid any damage to the equipment. 


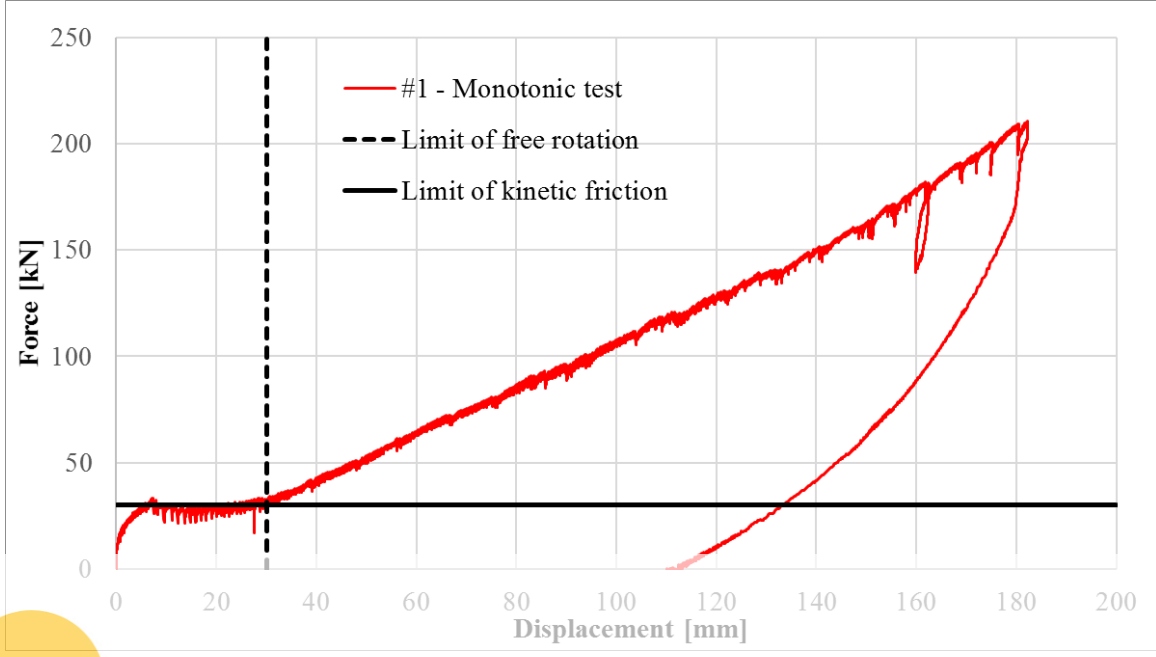

Figure 9: Specimens \#1 - Force-Displacement graph

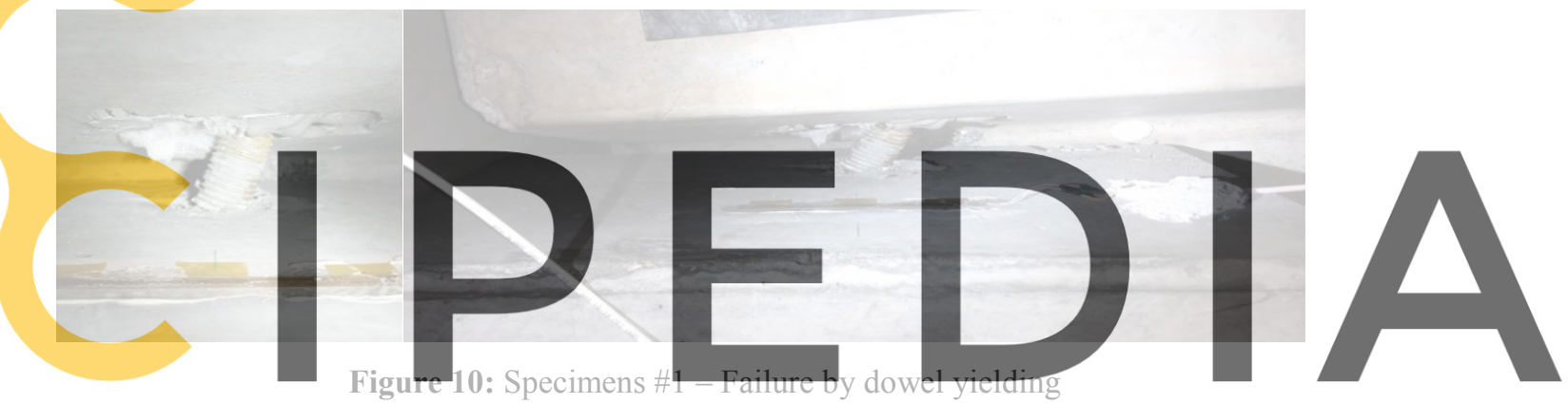

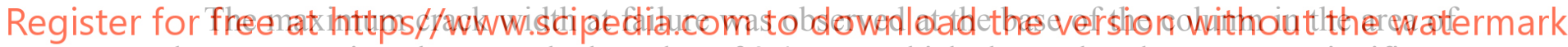
the cross-section change. It had a value of $0.45 \mathrm{~mm}$, which shows that there was no significant damage to the column and the failure of the assembly was strictly due to the connection failure.

\subsection{Results for test specimens \#2 - Cyclic test}

For the cyclic test the load displacement increments were chosen based on the initial monotonic test, the maximum displacement from the first test was divided into three increment groups: $60 \mathrm{~mm}, 120 \mathrm{~mm}$ and $180 \mathrm{~mm}$ (see Figure 7).

In compression, the whole strength of the assembly is larger than in tension because of the contact between the concrete elements (beam end and the column fork). Based on this assumption, it was expected that the failure will not occur in compression and the load cycles were chosen in such a way that the final step is under a tensioning load, which is the most unfavourable loading direction.

Failure point was reached in tension for a force value of $270 \mathrm{kN}$ with a maximum displacement of approximately $207 \mathrm{~mm}$ (Figure 11). It should be taken into consideration that the concrete used in these tests was high strength (around 70MPa compressive strength). In 
normal practice $\mathrm{C} 35 / 45$ is used, meaning that failure would occur even sooner.

Failure was a combination of steel dowel yielding and concrete spalling in the column fork, around the dowel (Figure 12). Concrete coverage of the dowel is insufficient for this particular connection. For this situation, the maximum crack width was slightly larger, $0.60 \mathrm{~mm}$, and in the same position as for the first test (base of the column in the area of crosssection change). This indicates that there is no significant damage to the column and the failure of the assembly was strictly related to the failure of the connection.

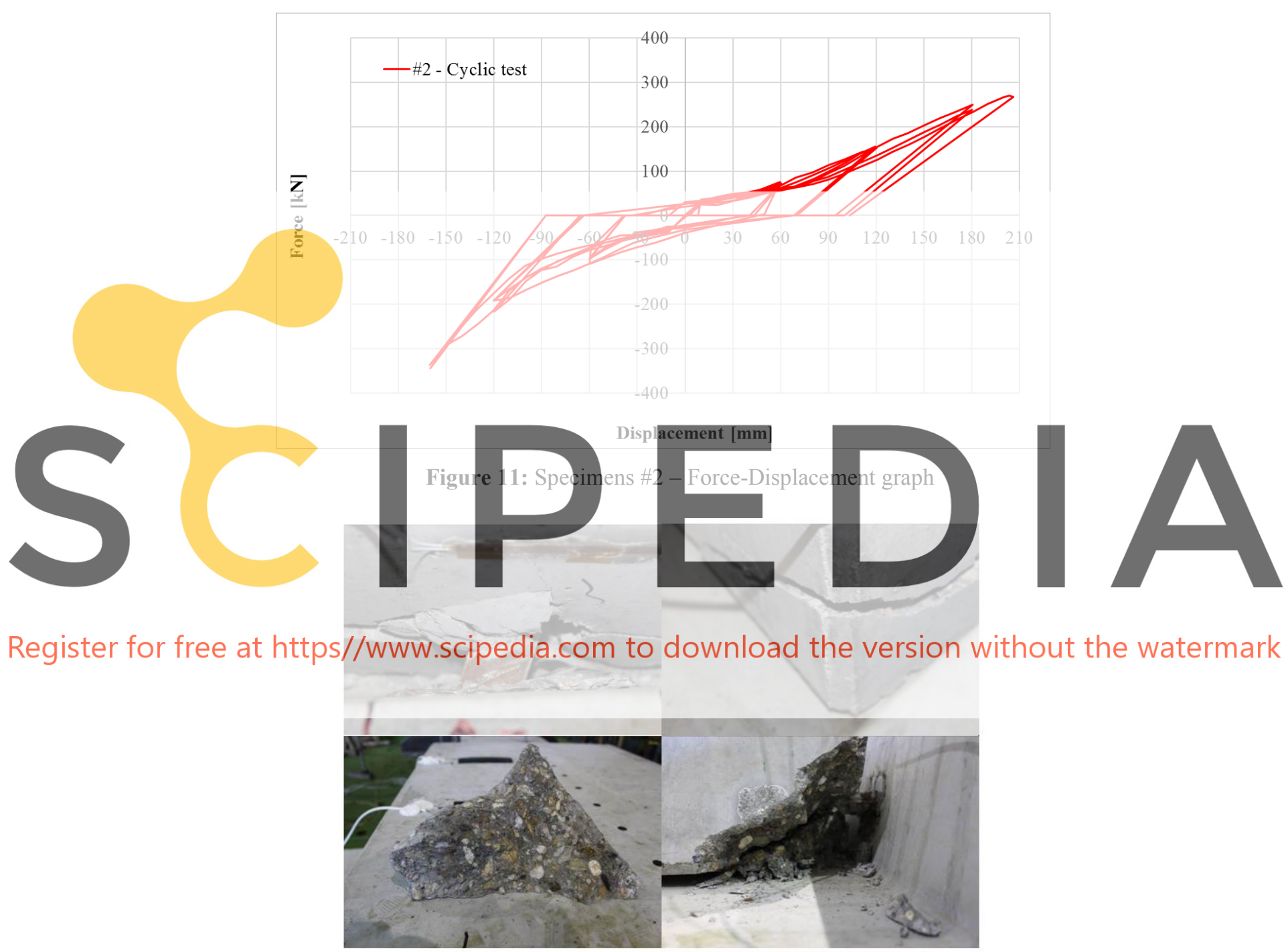

Figure 12: Specimens \#2 - Failure by dowel yielding and concrete spalling

\subsection{Results for test specimens \#3 - Cyclic test}

In this test, the beam web was larger, with a width increase from $18 \mathrm{~cm}$ to $30 \mathrm{~cm}$. This also reduces the thickness of the fork sides in the column. In this situation, the steel dowels and the sleeves inside the beam are also shifted, outwards, to the sides of the elements, resulting in a substantial reduction of concrete cover around these connection elements. 
Failure was reached in tension at $245 \mathrm{kN}$ with a maximum displacement of $210 \mathrm{~mm}$ (Figure 13). The failure mechanism was again a combination of the steel dowel yielding and concrete spalling (Figure 14). The smaller concrete cover for the dowels influences the capacity of this connection resulting in a smaller failure load compared to the previous cyclic test. The difference in maximum load can be seen also in the case of the maximum crack width at the base of the column, in the area of cross-section change. For this case the value was $0.50 \mathrm{~mm}$ compared to $0.60 \mathrm{~mm}$ for test specimens \#2. At the final step there was a large relative displacement between the column and the beam. The relative horizontal displacement between the beam and the column was approximately $6 \mathrm{~cm}$ at failure.

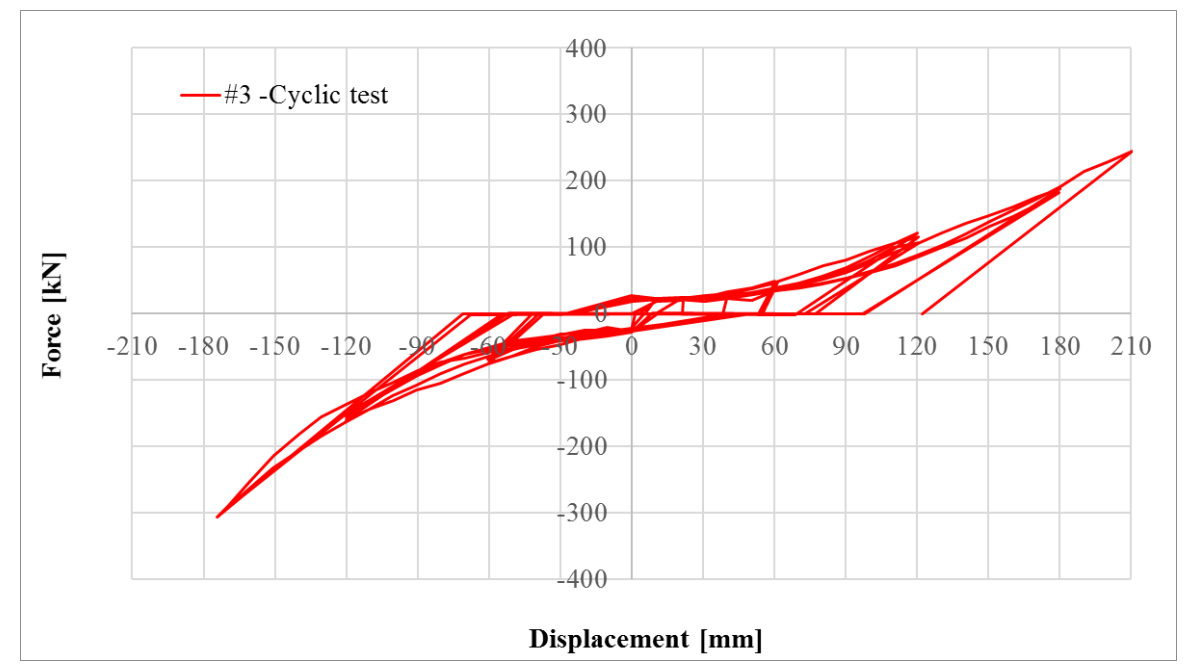

Figure 13: Specimens \#3 - Force-Displacement graph

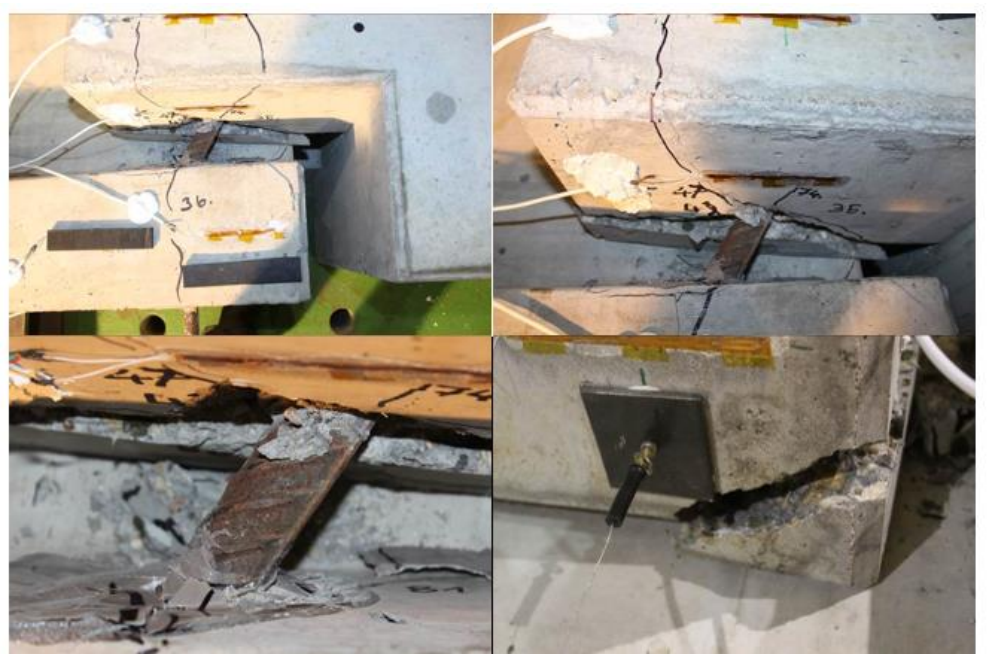

Figure 14: Specimens \#3 - Failure by dowel yielding and concrete spalling

\section{CONCLUSIONS}

- Tests have shown that these precast beam-columns connections are prone to ductile failure in the case of lateral loads. 
- According to basic seismic design rules these connections should fail after the formation of a dissipation mechanism at the base of the column. It is clear that the connections failed before the plastic hinge developed at the base of the column. In this situation the hypothesis that the connections remain in the elastic range during horizontal actions is not valid. Adequate formulas are needed for calculating these critical connections. The failure mechanism was either because of dowel yielding, concrete spalling around the dowel, or a combination of both.

- In the case of an earthquake, there is no significant energy dissipation in the concrete elements, and improper detailing of these connections may lead to partial or total failure of the joint, consequently followed by the collapse of the whole structure.

Acknowledgements. The financial support by CONSOLIS is greatly acknowledged. Special thanks to Wim Jansze and Gabriel Tarta for their unwavering support.

The research activities were supported by the project POCU/380/6/13/123927 ANTREDOC, "Entrepreneurial competencies and excellence research in doctoral and postdoctoral studies programs", project co-funded from the European Social Fund through the Human Capital Operational Program 2014-2020.

\section{REFERENCES}

[1] ELLIOTT, K. S.. Precast Concrete Structures. Boca Raton: Taylor \& Francis, CRC Press, (2017).

[2] Task Group 6.10 and PCI, fib Bulletin 78: Precast-concrete buildings in seismic areas, International Federation for Strucural Concrete (fib) (2016).

[3] Task Group 6.12: fib Design Handbook, Planing and design handbook on precast building structures. International Federation for Structural Concrete, Lausanne (2014).

[4] Savoia, M. Buratti, N. and Vincenzi, L. Damage and collapses in industrial precast buildings after the 2012 Emilia earthquake. Engineering Structures, vol. 137, p. 162180 (2017).

[5] Technical Committee CEN/TC 250 "Structural Eurocodes", Eurocode 8 : Design of structures for earthquake resistance - Part 1: General rules, seismic actions and rules for buildings. European Committee for Standardisation, Brussels (2003).

[6] European Comision, Design Guidelines for Connections of Precast Structures under Seismic Actions. Joint Research Centre of the European Commission, Luxembourg (2012).

[7] Zoubek, B. Isakovic, T. Fahjan Y. and Fischinger, M. Cyclic failure analysis of the beamto-column dowel connections in precast industrial buildings. Engineering Structures, vol. 52, p. 179-191 (2013).

[8] Sika Services AG, Product Data Sheet for Sika AnchorFix-3030. Zürich (2019).

[9] MSZ EN 12390-3:2015, Testing hardened concrete. Compressive strength of test specimens.

[10] MSZ EN 12390-13:2014, Testing hardened concrete. Determination of secant modulus of elasticity in compression.

[11] MSZ EN 12390-5:2009, Testing hardened concrete. Flexural strength of test specimens. 